\title{
Non-Mammalian Infants Requiring Parental Care Elicit Greater Human Caregiving Reactions Than Superprecocial Infants Do
}

\author{
Daniel J. Kruger
}

University of Michigan, Ann Arbor, MI, USA

\author{
Correspondence \\ Daniel J. Kruger, School of Public Health, \\ University of Michigan, 1420 Washington \\ Heights, Ann Arbor, MI 48109-2029. \\ E-mail: kruger@umich.edu \\ Received: December 31, 2014 \\ Initial acceptance: March 16, 2015 \\ Final acceptance: March 30, 2015 \\ (E. Hebets) \\ doi: $10.1111 /$ eth.12391
}

Keywords: caretaking, neoteny, parental care, pedomorphic, precocial, social cognition

\begin{abstract}
Ethologists proposed the coevolution of pedomorphic characteristics in infants and caregiving responses to these features in parents. Human infants higher in pedomorphic characteristics are more likely to receive baby talk from adults and elicit stronger motivations for caretaking. Neotenous facial characteristics in human adults cue social approach and elicit helping. This study demonstrates generally strong differences in reactions to infants from non-mammalian species requiring parental care (semiprecocial) and superprecocial species within the same class. People perceive semiprecocial infants as more neotenous (cute, immature, and helpless), had greater desires to hold or pet them, thought they would have a greater appreciation of being held or pet, thought they would be less likely to survive on their own, and anticipated being more likely to adopt them compared to superprecocial infants. Both avian and reptilian infants elicited these patterns. There was a moderate degree of sex differences in ratings, women saw the infants as more neotenous and were more willing to adopt them than men.
\end{abstract}

\section{Introduction}

Ethologist Konrad Lorenz (1943) proposed the coevolution of pedomorphic characteristics in infants and caregiving responses to these features in parents. Lorenz described the Kindchenschema (baby schema) as a set of physical features that are perceived to be cute, evoke a positive affective response, motivate physical affection, and elicit caretaking. These include a large head, high and protruding forehead, large eyes, small nose and mouth, etc. This system would be adaptive in species where infants require parental care (Bowlby 1969; Eibl-Eibesfeldt 1989). Fraley et al. (2005) found that among mammalian species, neoteny was associated with a cluster of characteristics including adult attachment, paternal care, small social groups, and small body sizes.

Large eyes, a large and protruding forehead, a small chin, pudgy lips, and thin, arched brows characterize youth in humans (Alley 1988). Baby schema features are prioritized by the human attention system (Brosch et al. 2007) and elicit perceptions of cuteness (Alley 1981; Glocker et al. 2009a), and infants with a greater degree of these features are more likely to receive baby talk from adults (Zebrowitz et al. 1992) and elicit stronger motivations for caretaking (Glocker et al. 2009a). Adult human faces with these features promote attributions of not only warmth, honesty, and sincerity but also naiveté and physical weakness (McArthur \& Apatow 1983; Berry 1991).

Human infant, human adult, and cat faces that were digitally modified to look more human infantlike were rated as cuter than those digitally modified to look more human adult-like (Little 2012). Keating et al. (2003) found that pictures of adult faces digitally modified with enlarged eyes and lips (neotenous features) were rated as more submissive, weak, naive, feminine, compassionate, and honest than the original image. When these pictures were added to fictitious resumes with stamped, addressed envelopes and left in public places, utilizing Milgram et al. (1965) lost letter technique, resumes with a neotenous image were more likely to be returned by mail than those with the original image. Keating et al. (2003) concluded that neotenous facial characteristics cue social approach and elicit helping. Supporting this notion, 
higher degrees of baby schema in digitally manipulated infant faces produce greater activation in the nucleus accumbens, which mediates reward processing and appetitive motivation, in nulliparous women (Glocker et al. 2009b).

The current study tests the notion that convergent evolution for infant pedomorphism across non-mammalian species exhibiting parental care will enhance the elicitation of caretaking reactions in humans. Lorenz (1943) described the similarities in neotenous features between species with parental care, although no previous research has demonstrated that across nonhuman species, such features enhance caretaking reactions. People should perceive infants from species requiring parental care as more cute and helpless than precocial species within the same class and should anticipate greater intentions to interact with and care for semialtricial than precocial infants. Because women on average are more interested in infants and caretaking activities than men are (Berman 1980; Maestripieri \& Pelka 2002), perceptions of neoteny and intentions to care may be higher in women than in men. Glocker et al. (2009b) found that women were more sensitive to infant cuteness than men were.

Gill (1995) classifies birds along a continuum from altricial to superprecocial, with semialtricial, semiprecocial, subprecocial, and precocial as intermediary categories. Gill (1995) defines superprecocial birds as those exhibiting no parental care; the young are completely independent at hatching. Female Megapodiidae, such as the Australian brush turkey, lay their eggs in a nest of decomposing vegetation and cover them with a layer of sand. The decomposing vegetation generates heat to incubate the eggs; males tend to the nest to regulate its temperature. Parents do not provide any care for the chicks, which are able to fly and fend for themselves soon after hatching. Blackheaded ducks are obligate brood parasites. Females lay their eggs in the nests of other birds; however, neither the chicks nor the adults destroy the eggs or kill the chicks of the host. Immediately after incubation, the ducklings fledge and are completely independent after a few hours, leaving their broodmates in the nest and fending for themselves.

In great contrast to superprecocial birds, altricial birds are naked, blind (their eyes are closed), and helpless at hatching. The characteristics of altricial bird infants are so extreme (and may even consist of a different mechanism) that they may not be suitable for generating a strong test of our hypothesis. Instead, we compare reactions to superprecocial bird infants with those in an intermediate category, semiprecocial bird infants. Gill (1995) defines semiprecocial birds as those whose young are somewhat mobile at hatching but remain with and are fed by their parents. Among the reptiles, only crocodiles and their relatives tend to both eggs and hatchlings (Ehrlich et al. 1988). Thus, an infant crocodile may elicit greater neoteny perceptions and caretaking intentions than an infant lizard, although neoteny and caretaking ratings for these reptiles may be lower than those for birds.

Hypotheses: (1) Ratings of neotenous features, attractive, cute, helpless, independent (R), mature (R), and young, will be higher for semiprecocial animal infants than for superprecocial animal infants. (2) Scores for caregiving reaction items (e.g., 'To what extent would you like to hold or pet this animal?') will be higher for semiprecocial animals than for superprecocial animals. 'How likely would this animal be able to survive on its own?' will exhibit the reverse pattern. (3) Women will have higher ratings of neotenous features and higher caregiving reactions than men across items.

\section{Method}

Google images were searched using a combination of each animal name (megapode, black-headed duck, tern, gull, penguin, crocodile, and lizard) and the terms 'hatchling', 'chick', and 'infant'. Images were selected based on the following criteria: (1) a highresolution color picture with no visual alterations, (2) depicting at least $80 \%$ of the animal, (3) including its entire head, (4) no portion of the animal 'off camera', (5) in its natural environment, and (6) by itself with no other animals visible. The first picture seen of each species that matched all six of these criteria was selected; images were cropped as necessary to remove any added text and resized so that animals were similar in pixel sizes on-screen. Most of the images were accompanied by descriptions on their webpages indicating that the animals had recently hatched.

Superprecocial bird images included the following: (1) Australian brush turkey, Alectura lathami (a megapode), (2) Tabon megapode, Megapodius cumingii; and (3) black-headed duck, Heteronetta atricapilla. Semiprecocial bird images included the following: (1) least tern, Sternula antillarum; (2) ring billed gull, Larus delawarensis; and (3) emperor penguin, Aptenodytes forsteri. Using the method described above, images were obtained for a dwarf crocodile, Osteolaemus tetraspis, and a superprecocial California alligator lizard, Elgaria multicarinata multicarinata.

Ethnically diverse undergraduates $(\mathrm{n}=172 ; 50 \%$ female, $M$ age $=19$, SD age $=1$ ) from a public university in the Midwestern United States completed anon- 
ymous online surveys at their convenience. Participants were asked to complete the surveys in locations where their responses would be private. Participants were presented with images of each animal followed by a standard series of questions, on separate pages in randomized order. Participants rated each image on items using a sliding scale ranging from 0 to $100 \%$ and initially set at $50 \%$. Selected values were displayed to participants, and each scale could be modified until participants advanced to the next page. Participants first rated each animal on the following terms: attractive, cute, helpless, independent, mature, and young, presented in randomized order. Participants then answered the following items: 'To what extent would you like to hold or pet this animal?', 'To what extent do you think this animal would appreciate being held or pet?', 'How likely would this animal be able to survive on its own?', and 'If you were working out in the field and found this animal wandering around by itself, how likely would you be to adopt it at least temporarily to make sure that it survived?' These items were followed by the item: 'Do you recognize this animal?' with the responses yes, maybe, and no. Those who responded 'yes' or 'maybe' were shown an additional question with open-ended text response: 'What is it? What do you know about it?' We also include an open-ended question for participant comments on each of the image pages.

All participants and data were included in analyses. Analyses assessed interitem reliability for the following neoteny items: attractive, cute, helpless, independent (reverse scored), mature (reverse scored), and young. Ratings for neoteny and the caretaking items (hold, be held, survive, and adopt) were examined by repeated-measures ANOVAs with linear contrasts comparing semiprecocial species to superprecocial species. Multilevel models, with ratings (Level 1) nested in participants, (Level 2) examined differences by participant sex, requirements for parental care (semiprecocial vs. superprecocial), class (bird vs. reptile), and interactions between (1) participant sex and requirements for parental care, and (2) participant sex and class.

\section{Results}

As predicted, ratings of neoteny (pedomorphic features) were higher for semiprecocial animals than for superprecocial animals (see Tables 1 and 2). The neoteny items had good or fair interitem reliability across species, except for the least tern (see Table 1). Participants had greater desire to hold or pet semiprecocial animals, thought that semiprecocial animals would have a greater appreciation of being held or pet, and anticipated being more likely to adopt semiprecocial animals compared to superprecocial animals, confirming predictions (see Table 2). There were large effects for ratings of neoteny, desire to hold or pet, and ability to survive and medium effects for presumed desire to be held or pet and willingness to adopt (see Cohen 1988).

Multilevel modeling replicated the differences by requirements for parental care (semiprecocial vs. superprecocial) for all outcomes (see Table 3). In addition, birds were rated higher in neoteny, higher in desire to hold or pet, higher in estimated appreciation

Table 1: Descriptive results

\begin{tabular}{|c|c|c|c|c|c|c|}
\hline Species & $\begin{array}{l}\text { Neoteny } \\
\text { Alpha }\end{array}$ & $\begin{array}{l}\text { Neoteny } \\
(M, 95 \% \mathrm{Cl})\end{array}$ & $\begin{array}{l}\text { Hold } \\
(\mathrm{M}, 95 \% \mathrm{Cl})\end{array}$ & $\begin{array}{l}\text { Be held } \\
(M, 95 \% \mathrm{Cl})\end{array}$ & $\begin{array}{l}\text { Survive } \\
(M, 95 \% \mathrm{Cl})\end{array}$ & $\begin{array}{l}\text { Adopt } \\
(M, 95 \% \mathrm{Cl})\end{array}$ \\
\hline Least tern & 0.445 & $\begin{array}{l}72.96 \\
71.11-74.83\end{array}$ & $\begin{array}{l}65.23 \\
60.35-70.11\end{array}$ & $\begin{array}{l}39.59 \\
35.44-43.75\end{array}$ & $\begin{array}{l}22.85 \\
19.66-26.04\end{array}$ & $\begin{array}{l}47.38 \\
42.51-52.26\end{array}$ \\
\hline Ring billed gull & 0.658 & $\begin{array}{l}59.45 \\
56.99-61.91\end{array}$ & $\begin{array}{l}40.99 \\
35.79-46.19\end{array}$ & $\begin{array}{l}26.28 \\
22.35-30.21\end{array}$ & $\begin{array}{l}30.52 \\
26.84-34.20\end{array}$ & $\begin{array}{l}32.38 \\
27.63-37.14\end{array}$ \\
\hline Dwarf crocodile & 0.799 & $\begin{array}{l}45.29 \\
42.25-48.33\end{array}$ & $\begin{array}{l}29.48 \\
24.30-34.66\end{array}$ & $\begin{array}{l}17.50 \\
14.26-20.74\end{array}$ & $\begin{array}{l}54.30 \\
49.93-58.67\end{array}$ & $\begin{array}{l}22.56 \\
18.14-26.98\end{array}$ \\
\hline Australian brush turkey & 0.789 & $\begin{array}{l}44.18 \\
41.34-47.02\end{array}$ & $\begin{array}{l}26.98 \\
22.62-31.33\end{array}$ & $\begin{array}{l}21.16 \\
17.62-24.71\end{array}$ & $\begin{array}{l}46.92 \\
42.68-51.16\end{array}$ & $\begin{array}{l}25.99 \\
21.56-30.42\end{array}$ \\
\hline Tabon megapode & 0.787 & $\begin{array}{l}40.15 \\
37.29-43.01\end{array}$ & $\begin{array}{l}30.93 \\
26.35-35.51\end{array}$ & $\begin{array}{l}20.99 \\
17.50-24.47\end{array}$ & $\begin{array}{l}56.16 \\
51.86-60.46\end{array}$ & $\begin{array}{l}28.31 \\
23.68-32.94\end{array}$ \\
\hline California alligator lizard & 0.717 & $\begin{array}{l}25.57 \\
23.40-27.74\end{array}$ & $\begin{array}{l}17.97 \\
13.92-22.01\end{array}$ & $\begin{array}{l}11.34 \\
8.71-13.96\end{array}$ & $\begin{array}{l}66.28 \\
61.77-70.79\end{array}$ & $\begin{array}{l}15.70 \\
11.91-19.48\end{array}$ \\
\hline
\end{tabular}


Table 2: Results of repeated-measures ANOVAs and linear contrasts

\begin{tabular}{lcccccc}
\hline & \multicolumn{2}{l}{ Omnibus test } & & \multicolumn{2}{l}{ Contrast } \\
\cline { 2 - 3 } \cline { 6 - 7 } & $F_{(1,1197)}$ & $p$ & & $F_{(1,1197)}$ & $p$ & $\eta^{2}$ \\
\hline Neoteny & 224.22 & 0.0001 & & 1039.91 & 0.0001 & 0.372 \\
Hold & 159.47 & 0.0001 & & 532.50 & 0.0001 & 0.155 \\
Be held & 66.39 & 0.0001 & & 123.51 & 0.0001 & 0.091 \\
Survive & 76.52 & 0.0001 & & 339.89 & 0.0001 & 0.213 \\
Adopt & 211.25 & 0.0001 & & 130.18 & 0.0001 & 0.075 \\
\hline
\end{tabular}

of being held or pet, less likely to survive on their own, and more likely to be adopted than reptiles were. Women gave higher ratings for neoteny and willingness to adopt than men. Participant sex moderated differences in ratings. Women exhibited greater differences by class than men, with even lower ratings for reptiles on neoteny, desire to hold or pet, and willingness to adopt compared to men's ratings. Women also showed a stronger discrimination between semiprecocial and superprecocial species on estimated appreciation of being held or pet, compared to men.

Participants were most likely to recognize the emperor penguin as a 'penguin' (57\%, 6 specified emperor penguin), $42 \%$ identified the black-headed duck as a 'duck' (none as a black-headed duck), 24\% identified the California alligator lizard as a 'lizard' (none as a California alligator lizard), 16\% identified the dwarf crocodile as a 'crocodile' (none as a dwarf crocodile), two participants identified the least tern, two participants identified the Australian brush turkey as a 'turkey', and no participants identified the ring billed gull or Tabon megapode.

\section{Discussion}

The current results confirmed the predictions that people would ( 1 ) perceive infants from species requiring parental care as more neotenous (cute, immature, and helpless) than precocial species within the same class and (2) anticipate greater intentions to interact with and care for semiprecocial than superprecocial infants. Women saw the infants as more neotenous and were more willing to adopt them than men. The results supported the hypothesis that convergent evolution for infant pedomorphism across non-human species exhibiting parental care enhances the elicitation of caretaking reactions in humans. The enhancement of caregiving reactions by species with parental care was evident for both bird and reptile infants, although bird infants elicited greater caregiving responses on average than reptile infants. Responses to the semiprecocial dwarf crocodile were similar to those for the superprecocial birds. Overall, this study elaborates the understanding of psychological dynamics related to pedomorphism and caretaking. The results suggest similarity in care-eliciting features and motivating mechanisms across a wide range of species.

These findings complement results from studies using phylogenetic analyses of physical morphology among mammalian species (e.g., Fraley et al. 2005), human infants as stimuli (e.g., Alley 1981; Zebrowitz et al. 1992), and computer manipulations of images (e.g., Glocker et al. 2009a,b; Little 2012). It is notable that women did perceive infants as more cute, immature, and helpless and had higher intentions to care for these infants if found on their own, but did not have greater desires to hold or pet or perceive these infants as more appreciative of being held or pet. This suggests that sex differences in motivations to care for such non-mammalian infants are not based on hedonistic or self-serving desires, but perhaps greater feelings of concern or responsibility. Future research may clarify this issue. It is also notable that across species, participants (on average) recognized that that their desires to hold or pet these infants were not matched by the infants' own desires to be pet or held. Some participants even noted this explicitly in their comments.

As with any study, there are limitations. The participants are US undergraduates; such samples have been criticized as not being representative of the entire human population (e.g., Henrich et al. 2010). It is not known how these reactions may be shaped by the extent of experiences with the natural world and/or contact with non-domesticated animals. The stimuli were selected from publicly available images from different sources, so they were not standardized (as computer-generated images could be), in viewing angle, lighting, background, stance and posture, or other properties that may influence perceptions and reactions. Nor were the images geometrically assessed or manipulated for the degree of neoteny, computergenerated images could be given precise dimensions. The image limitations are a trade-off for the use of natural, real world stimuli. Although computer-generated images have the advantage of consistency and control, they can also be unrealistic, for example including supernormal stimuli outside of the natural range of attributes.

Familiarity is associated with liking (Zajonc 2001), and the penguin was the most familiar animal, consistent with its frequent portrayal in entertainment and educational media. However, the next two most often identified animals were superprecocial and very few individuals identified any of the others. Thus, famil- 
Table 3: Results of multilevel models

\begin{tabular}{|c|c|c|c|c|c|c|}
\hline Fixed Effects & & Coefficient & SE & $t$ & $d f$ & $p$ \\
\hline \multicolumn{7}{|l|}{ Neoteny scale } \\
\hline \multirow[t]{2}{*}{ For $\beta_{0 j}$ Intercept } & Intercept, $\gamma_{0 o}$ & 33.26 & 1.92 & 17.32 & 170 & 0.004 \\
\hline & Sex, $\gamma_{01}$ & 10.04 & 3.84 & 2.61 & 170 & 0.010 \\
\hline \multirow[t]{2}{*}{ For $\beta_{1 j}$ Precocial slope } & Intercept, $\gamma_{10}$ & 26.14 & 0.85 & 30.68 & 1370 & 0.001 \\
\hline & Sex, $\gamma_{11}$ & -2.55 & 1.70 & -1.50 & 1370 & 0.134 \\
\hline \multirow[t]{5}{*}{ For $\beta_{2 j}$ Class slope } & Intercept, $\gamma_{20}$ & -18.52 & 0.98 & -18.82 & 1370 & 0.001 \\
\hline & Sex, $\gamma_{21}$ & -4.76 & 1.97 & -2.42 & 1370 & 0.016 \\
\hline & Random effect & $S D$ & var. & $\chi^{2}$ & $d f$ & $p$ \\
\hline & Intercept, $u_{0}$ & 7.86 & 61.76 & 506.23 & 170 & 0.001 \\
\hline & Level 1 & 15.80 & 249.73 & & & \\
\hline \multicolumn{7}{|l|}{ Desire to hold or pet } \\
\hline \multirow[t]{2}{*}{ For $\beta_{0 j}$ Intercept } & Intercept, $\gamma_{0 o}$ & 26.88 & 3.27 & 8.21 & 170 & 0.001 \\
\hline & Sex, $\gamma_{01}$ & 7.34 & 6.55 & 1.12 & 170 & 0.264 \\
\hline \multirow[t]{2}{*}{ For $\beta_{1 j}$ Precocial slope } & Intercept, $\gamma_{10}$ & 26.83 & 1.30 & 20.63 & 1370 & 0.001 \\
\hline & Sex,$\gamma_{11}$ & 4.77 & 2.60 & 1.83 & 1370 & 0.067 \\
\hline \multirow[t]{5}{*}{ For $\beta_{2 j}$ Class slope } & Intercept, $\gamma_{20}$ & -21.70 & 1.50 & -14.45 & 1370 & 0.141 \\
\hline & Sex, $\gamma_{21}$ & -9.81 & 3.00 & -3.27 & 1370 & 0.002 \\
\hline & Random effect & $S D$ & var. & $\chi^{2}$ & $d f$ & $p$ \\
\hline & Intercept, $u_{0}$ & 22.58 & 509.72 & 1361.07 & 170 & 0.001 \\
\hline & Level 1 & 24.12 & 581.98 & & & \\
\hline \multicolumn{7}{|c|}{ Estimated appreciation of being held or pet } \\
\hline \multirow[t]{2}{*}{ For $\beta_{0 j}$ Intercept } & Intercept, $\gamma_{00}$ & 22.65 & 2.43 & 9.31 & 170 & 0.001 \\
\hline & Sex, $\gamma_{01}$ & -7.68 & 4.87 & -1.58 & 170 & 0.116 \\
\hline \multirow[t]{2}{*}{ For $\beta_{1 j}$ Precocial slope } & Intercept, $\gamma_{10}$ & 12.22 & 0.99 & 12.34 & 1370 & 0.001 \\
\hline & Sex, $\gamma_{11}$ & 6.77 & 1.98 & 3.42 & 1370 & 0.001 \\
\hline \multirow[t]{5}{*}{ For $\beta_{2 j}$ Class slope } & Intercept, $\gamma_{20}$ & -13.28 & 1.14 & -11.62 & 1370 & 0.001 \\
\hline & Sex, $\gamma_{21}$ & -0.37 & 2.29 & -0.161 & 1370 & 0.873 \\
\hline & Random effect & $S D$ & var. & $\chi^{2}$ & $d f$ & $p$ \\
\hline & Intercept, $u_{0}$ & 15.66 & 245.09 & 1157.74 & 170 & 0.001 \\
\hline & Level 1 & 18.37 & 337.43 & & & \\
\hline \multicolumn{7}{|l|}{ Ability to survive } \\
\hline \multirow[t]{2}{*}{ For $\beta_{0 j}$ Intercept } & Intercept, $\gamma_{00}$ & 52.77 & 3.24 & 16.29 & 170 & 0.001 \\
\hline & Sex, $\gamma_{01}$ & 0.70 & 6.48 & 0.108 & 170 & 0.915 \\
\hline \multirow[t]{2}{*}{ For $\beta_{1 j}$ Precocial slope } & Intercept, $\gamma_{10}$ & -15.55 & 1.41 & -10.96 & 1370 & 0.001 \\
\hline & Sex,$\gamma_{11}$ & 1.39 & 2.84 & 0.49 & 1370 & 0.623 \\
\hline \multirow[t]{5}{*}{ For $\beta_{2 j}$ Class slope } & Intercept, $\gamma_{20}$ & 15.43 & 1.71 & 9.01 & 1370 & 0.001 \\
\hline & Sex, $\gamma_{21}$ & 3.60 & 3.28 & 1.10 & 1370 & 0.272 \\
\hline & Random effect & $S D$ & var. & $\chi^{2}$ & $d f$ & p \\
\hline & Intercept, $u_{0}$ & 14.71 & 216.26 & 594.43 & 170 & 0.001 \\
\hline & Level 1 & 26.32 & 692.82 & & & \\
\hline \multicolumn{7}{|l|}{ Willingness to adopt } \\
\hline \multirow[t]{2}{*}{ For $\beta_{0 \mathrm{j}}$ Intercept } & Intercept, $\gamma_{0 o}$ & 30.88 & 3.31 & 9.31 & 170 & 0.001 \\
\hline & Sex, $\gamma_{01}$ & 11.71 & 6.64 & 1.76 & 170 & 0.041 \\
\hline \multirow[t]{2}{*}{ For $\beta_{1 j}$ Precocial slope } & Intercept, $\gamma_{10}$ & 11.34 & 1.15 & 9.88 & 1370 & 0.001 \\
\hline & Sex, $\gamma_{11}$ & 0.23 & 2.29 & 0.10 & 1370 & 0.913 \\
\hline \multirow[t]{5}{*}{ For $\beta_{2 j}$ Class slope } & Intercept, $\gamma_{20}$ & -14.38 & 1.38 & -10.44 & 1370 & 0.001 \\
\hline & Sex, $\gamma_{21}$ & -6.20 & 2.75 & -2.25 & 1370 & 0.012 \\
\hline & Random effect & $S D$ & var. & $\chi^{2}$ & $d f$ & $p$ \\
\hline & Intercept, $u_{0}$ & 22.69 & 514.79 & 1983.54 & 170 & 0.001 \\
\hline & Level 1 & 19.65 & 386.04 & & & \\
\hline
\end{tabular}

iarity is unlikely to be confounded for the range of findings. Finally, this study assessed perceptions and hypothetical reactions, not the actual behaviors of individuals encountering non-mammalian infants.
In conclusion, this is the first study to demonstrate differences in human reactions to non-mammalian infants based on requirements for parental care in each species. Thus, this study provides the first 
cross-species (and cross-class) empirical evidence for Lorenz (1943) proposal for the convergent evolution of neotenous features eliciting caregiving responses. Importantly, the neotenous characteristics eliciting care are linked to the actual dependency of the infant and thus a convergent adaptation (Bowlby 1969; EiblEibesfeldt 1989).

\section{Acknowledgements}

The author is grateful to Cindy Hazan and Scott Harber for helpful feedback and to Cornell's Adult University for a stimulating intellectual environment.

\section{Literature Cited}

Alley, T. R. 1981: Head shape and the perception of cuteness. Dev. Psychol. 17, 650-654.

Alley, T. R. 1988: The effects of growth and aging on facial aesthetics. In: Social and Applied Aspects of Perceiving Faces. (Alley, T. R., ed). Erlbaum, Hillsdale, New Jersey, pp. 51-62.

Berman, P. W. 1980: Are women more responsive than men to the young? A review of developmental and situational variables. Psychol. Bul. 88, 668-695.

Berry, D. S. 1991: Attractive faces are not all created equal: joint effects of facial babyishness and attractiveness on social perception. Pers. Soc. Psychol. Bul 17, 523-533.

Bowlby, J. 1969: Attachment and Loss, Vol. 1. Basic Books, New York.

Brosch, T., Sander, D. \& Scherer, K. R. 2007: That baby caught my eye.. attention capture by infant faces. Emotion 7, 685-689.

Cohen, J. 1988: Statistical Power Analysis for the Behavioral Sciences, 2nd edn. Academic Press, New York.

Ehrlich, P., Dobkin, D. \& Wheye, D. 1988: The Birder's Handbook. Simon \& Schuster, New York.

Eibl-Eibesfeldt, I. 1989: Human Ethology. De Gruyter, New York.
Fraley, R. C., Brumbaugh, C. C. \& Marks, M. J. 2005: The evolution and function of adult attachment: a comparative and phylogenetic analysis. J. Pers. Soc. Psychol. 89, $731-746$.

Gill, F. B. 1995: Ornithology, 2nd edn. W.H. Freeman, New York.

Glocker, M. L., Gur, R. C., Langleben, D. D., Loughead, J. W., Ruparel, K. \& Sachser, N. 2009a: Baby schema in infant faces induces cuteness perception and motivation for caretaking in adults. Ethology 115, 257-263.

Glocker, M. L., Langleben, D. D., Ruparel, K., Loughead, J. W., Valdez, J. N., Griffin, M. D., Sachser, N. \& Gur, R. C. 2009b: Baby schema modulates the brain reward system in nulliparous women. Proc. Natl Acad. Sci. USA 106, 9115-9119.

Henrich, J., Heine, S. J. \& Norenzayan, A. 2010: The weirdest people in the world? Behav. Brain Sci 33, 6183.

Keating, C. F., Randall, D. W., Kendrick, T. \& Gutshall, K. A. 2003: Do babyfaced adults receive more help? The (Cross-Cultural) Case of the Lost Resume. J. Nonverbal Behav. 27, 89-109.

Little, A. C. 2012: Manipulation of infant-like traits affects perceived cuteness of infant, adult and cat faces. Ethology 118, 775-782.

Lorenz, K. 1943: Die angeborenen Formen moglicher Erfahrung. Z Tierpsychol 5, 234-409.

Maestripieri, D. \& Pelka, S. 2002: Sex differences in interest in infants across the lifespan: a biological adaptation for parenting? Hum. Nat. 13, 327-344.

McArthur, L. Z. \& Apatow, K. 1983: Impressions of babyfaced adults. Soc. Cognition 2, 315-342.

Milgram, S., Mann, L. \& Harter, S. 1965: The lost-lettertechnique: a tool of social research. Public Opin. Quart. 29, 437-438.

Zajonc, R. B. 2001: Mere exposure: a gateway to the subliminal. Curr. Dir. Psychol. Sci. 10, 224-228.

Zebrowitz, L. A., Brownlow, S. \& Olson, K. 1992: Baby talk to the babyfaced. J. Nonverbal Behav. 16, 143-158. 